\title{
Comparative analysis of energy based optimized dynamic source multipath routing protocol in WSNs
}

\author{
Nabeena Ameen, D. Najumnissa Jamal, L. Arun Raj \\ B.S.Abdur Rahman Crescent Institute of Science and Technology, India
}

\begin{tabular}{l} 
Article Info \\
\hline Article history: \\
Received Jan 28, 2019 \\
Revised Apr 5, 2019 \\
Accepted May 17, 2019 \\
\hline
\end{tabular}

\section{Keywords:}

Routing optimization Ant colony Optimization (ACO) Wireless Sensor Networks Energy efficient Routing protocols Packet Delivery

\begin{abstract}
Wireless Sensor Network (WSN) has been considered to be a formula for overcoming various requirements like surveillance, health care, traffic surveillance and defense systems. As an aid to the demands for Wireless sensor network, research has been going on the efficient consumption of energy and secure communication areas for WSN. Here introducing Ant colony optimization based Optimized dynamic Secure multipath routing protocol (ODMRP) have improvised WSN in terms of energy efficiency, performance, strength and scalability. This paper proposed mechanism of Ant Colony Optimization based on the optimized dynamic secure multipath routing protocol (ODMRP-ACO) for data transmission in the WSNs. This eases the data transmission via WSNs. The shortest path between the source node and the destination node is analyzed by the ant colony cooperation which is based on the ACO. There are several routing protocols like Energy-aware and Secure Routing with Trust (ESRT-ACO), Split multipath routing protocol-Ant colony Optimization (SMR-ACO), Light Weight Trust Based routing protocol (TLBAODV) and AODV routing protocols that are built with the ACO based Routing Protocol. They are concerned over the End to End Delay, Packet Delivery Rate, Routing Overhead, Throughput and Energy Efficiency. The data analytic results have proven to be effective compared to the other routing protocols designed for WSN. This is because; the overall ODMRP-ACO was calculated to be $96.98 \%$ better than the other existing. For the newly developed ODMRP-ACO protocol, the simulation platform used is NS2 simulation software.
\end{abstract}

Copyright () 2019 Institute of Advanced Engineering and Science. All rights reserved.

\section{Corresponding Author:}

Nabeena Ameen,

B.S.Abdur Rahman Crescent Institute of Science and Technology,

GST Road, Vandalur, Chennai 600 048,

Tamilnadu, India.

Email: nabeena@crescent.education

\section{INTRODUCTION}

Background: One of the newest technologies which are in great usage and demand is the wireless sensor network technology. Users come up with enormous data to store, retrieve and share with security. In, a huge network of remote servers is connected over the Internet. This is to store, share, manipulate, retrieve and process the data avoiding the interference of a local server or personal computer. Working from any place and any time is one among the advantages. The clients do not need to purchase any resource for their data storage [1,2]. The WSN has to increase its battery power so as to increase node's efficiency. A high performance computing for the WSN data storage is required. This is where WSN real usage is seen.

Problem: In computing overcomes the issues with a fairly low budget. Integrating WSN with protocol paves way for a flexible monitoring and data storage from WSN. A safer and more secure communication with energy-efficiency remains to be in open research for the WSN. Having low memory, restricted battery power, 
less computation capability and a small communication speed range, the wireless sensor nodes seek improvement. For transmitting the incoming packets, there arose the need for an energy efficient routing protocol. For this, the protocols based on ACO are equipped with better WSNs in the energy efficiency, performance, strength, network lifetime and scalability aspects. A reliable data communication with high speed and access for the consumers is to be managed very efficiently especially in health care applications.

Proposed solution: For the data transmission through WSN an optimized dynamic secure multipath routing protocol (ODMRP-ACO) based on Ant Colony Optimization is used. This finds the shortest path between the source and the destination nodes. Previously, when WSN was in practice, Energy-aware and Secure Routing with Trust (ESRT-ACO), Split multipath routing protocol-Ant colony Optimization (SMRACO), Light Weight Trust Based routing protocol (TLB-AODV) had limited processing power with minimal battery life. The communication speed was also low.

The best path between the source and the destination nodes is obtained by the Ant Colony Optimization. This method is based on the optimized dynamic secure multi-path routing protocol. Multiple paths are obtained on distance and energy basis is used by this protocol in order to deliver the data from source to destination. Selecting the path with maximum energy and minimum hop count within the group reduces the load on wireless nodes [3-5]. There are some phases which are considered to be milestones during the research. They are namely, node deployed in WSN, node identification, route selection, route maintained, ACO based dynamic multi-path selection based on energy, distance for data transmission and acknowledgement received. The simulation results have proven that the proposed protocol shows better performance when compared to existing ones. The existing protocols are, Energy-aware and Secure Routing with Trust (ESRT-ACO), Split multipath routing protocol-Ant colony Optimization (SMR-ACO), Light Weight Trust Based routing protocol (TLB-AODV) and AODV routing protocols. Performance based on minimum energy consumption, Maximize throughput, delay, packet delivery ratio and network lifetime has been improved.

The paper has the following sections: Section II and III explain the background and Related Work and Existing Wireless Sensor Network System. Section III explains the Proposed Method for Data transmitted in routing with WSN. Section IV provides the results and discussion with the conclusion given on Section V.

\section{BACKGROUND}

\subsection{Overviews in WSN}

WSN contains nodes that have low power, low cost that are capable of doing many functions. They are deployed in the regions of interest [6]. Being small in size, all the sensor nodes are embedded with sensors, microprocessors and radio transceivers which have sensing, data processing and communication abilities [7]. In comparison with the wireless communication networks that were used long back, like MANET and the cellular systems, there are some distinct features in the sensor nodes like deployed sensor nodes, battery powered sensor nodes, ability to self configurable. They have to be application specific, unreliable sensor nodes. Network topology changes and partitions are fed in WSN to prevent the environmental obstructions and node failure. like Environmental applications, Health applications, Military applications, Home application, Commercial applications etc are the fields where WSN's usage is seen.

\subsection{Routing issues}

Infrastructure less: The sensor nodes in wireless sensor networks are put up in a random infrastructure less way. Therefore, designing the routing protocols should be done in a very careful manner.

Energy constraints: Battery power is a main factor in sensor nodes. Care should be taken during the usage of available energy as batteries cannot be replaced.

Network lifetime: Batteries are the main reasons for the sensor node's lifetime. The stumpy energy node has to be determined once a node wishes to send the data. Network partitions may have to be done during the selection of stumpy energy. Therefore, to extend the network span, a node with balanced energy should be chosen [8].

Cost: The node's cost must be kept at low rate as there exists thousands of sensor nodes in the sensing area.

Scalability: The size of the network will be increasing and the sensor network should be capable of adapting to the change as the nodes may join new networks.

QoS: There are certain properties that the sensor network should have. They are, minimum delay, less control overhead, high throughput and efficient resource allocation [9].

Coverage: The sensing node has range, location and density based on which the coverage is determined. So it should be high. 


\section{RELATED WORK}

There are various challenges that has to be faced in Wireless Sensor Networks related to the Energy Efficiency, Limited Computational capability, Routing Overhead, Packet Delivery etc. It is always a tedious task to design Energy Efficient Routing Protocol for Wireless Sensor Networks. Swarm Intelligence based routing protocols are being introduced till now for overcoming the issues. The study of combined behavior of several systems with varied component designs is the idea about Swarm Intelligence. This is to have coordination among them through self-organization and decentralized controls. Those algorithms that are based on Swarm Intelligence and nature based intelligence produce good results and they are adaptive. A good platform for researches is provided by the Anand Nayyar [10] analysis and survey of Ant Colony Optimization based routing protocols. They can work over the faults and drawbacks of the protocols developed till now for coming up with a better protocol.

A very common research topic in the wireless sensor network (WSN) domain is the Routing Protocol for WSN in reference to the unsolved issues. Multicast routing protocols in the WSN and the various forms of standard research contribution along with significant new research techniques for bringing up the performance is the main discussion of the paper [11]. A novel architecture for explaining the optimization as a cost cutting issue regarding the multicast routing in WSN has been proposed to bridge the research gap. The current way of multicast routing performance in WSN is explained in this paper which will assist the readers on the possible direction of future with a very crisp display of the system's architecture. A study related to the energy dissipation by the use of multicast routing in sensor networks has been presented by Hao et al. The demanded characteristics for saving energy has also been discussed by the author which has been evaluated with respect to the network's span $[12,13]$.

A new multicast routing protocol technique has been introduced by Maddali [14] which follows distributed mechanism using various routing states. Heterogeneous routing was the formal beneficiary of this technique and a new node named as core node has been used. Factors like delay, latency, energy conservation factor, throughput, delay, packet delivery ratio, and overhead were used for testing the outcome of the study. In accordance with the ratio of residual energy of each node and the average energy of the network, the CHs are chosen in Single-hop Energy Efficient Clustering Protocol (S-EECP). Higher weighted nodes have increased chances of being selected as CHs. Data from CHs to the base station is transferred trough multi-hop communication in the Multi-hop Energy Efficient Clustering Protocol (M-EECP) [15].

For the data collection in WSNs, Velmani and Karthick have come up with a Velocity Energy-efficient and Link-aware Cluster-Tree (VELCT) scheme. The issues like distance coverage, mobility, delay, traffic, tree intensity and end-to-end connection are successfully reduced by VELCT. The node that collects data present in the DCT collects data packets from the cluster head and delivers them to the sink. It does not participate in information sensing. Minimal energy exploitation and reduced end-to-end delay and cluster head traffic in the WSNs are overcome by the VELCT scheme and the proper usage of the DCT [16].

A paper on 'attacks and counter measures' (Intrusion Detection Systems) was proposed by Anand Motwani et al. [17] for WSNs. This has given suggestions over the Optimized AODV Routing with "Ant based" technique which is for better security of WSNs saving them from sinkholes. Certain properties of the WSN routing protocol for crafting a secured and reliable WSN were analyzed by this work. The popular Network simulator versions (ns-2.33) is used for simulation.

The capacities of the network nodes are minimal in accordance with the energy supply, computational capacity restrictions and bandwidth of communication. This was reviewed by Seema Pahal et al. [18] on the WSN networks. The maintenance of routes present in the network is monitored by the wireless sensor networks which make sure of trusting multi-hop communication. Several protocols like Dynamic Source Routing (DSR), Dynamic MANET On-demand Protocol (DYMO), Zone Routing Protocol (ZRP) and Optimized Link-State Routing (OLSR) have been dealt with comparative factors like Residual Battery Capacity, Throughput, Average End-to-End Delay and Packet-Delay.

The Energy-aware sink relocation method (EASR) that follows the Energy-aware routing MCP was introduced by C. F. Wang et al. [19]. This routing method provides enhanced node supplement to the message relaying Sink node for avoiding user nodes that are not authorized. For maximizing the span of WSNs, Davut Incebacak et al [20] has proposed the Data compression methods which will look over the contextual privacy measures with the help of Mathematical programming frameworks. This saves energy than the static compression/decompression of independently compressed data. It is stated by Keontaek Lee et al. [21] that the consumption of energy purely depends on the traffic volume and that the target span has no guarantee through only energy-efficient routings. The rate of sensing and selection of routing has been optimized by the author. In several network configurations, the proposed schemes has attained near optimality that does not work over rate of sensing and selection of route. Because of the data relaying from several areas of the network towards the sink, there exists energy imbalance in the WSN. Subir Halder et al. [22] has claimed that malicious jammers are in practice for data sensing and transmission to the base station by means of multi-hop communication. 
Also, the sensors' usage is seen only for communication. In order to maximize the span of the network, duty scheduling of activities based on sensors is done in WSNs.

Sheikholeslami et al. [23] states that certain Quality of Service (QoS) is required by multimedia applications. For multimedia transmissions within the WSNs, an optimized routing based on the Ant Colony Optimization is proposed in this paper. An average throughput of $164.65 \mathrm{kbps}$ for multimedia data transmission can be obtained by optimized routing and a better QoS compared to DSDV and AODV routing protocols is achieved [24]. There are four major schemes in the WSN routing protocol. They are as follows: Network Structure, Communication Model, Topology, and Reliable Routing. The Ant Colony Optimization (ACO) is used in the new communication protocol [25] called the Energy- Efficient Ant-Based Routing Algorithm (EEABR) designed for WSN. For video transmissions in the WSNs, a cross-layer approach with the base on EDCA and H.264/SVC is being used. A minimized high throughput with packet overhead is ensured by the Real-time routing protocol with load distribution (RTLD) [26]. This extrapolates the span of the WSN. However, only constant bit rate (CBR) traffic is used by this routing protocol in the simulation program.

In order to develop an effective routing enhancing the MWSNs' lifetime, the Dominated Sorting Genetic Algorithm-II based Routing Protocol [27] (NSGAII-RP) was introduced. This protocol looks into the routing issues as well as the coverage of it. The NSGAII algorithm is used to optimize the coordinates of the mobile nodes so as to find the optimized $\mathrm{CHs}$ on energy consumption basis and network coverage. For improving the span of MWSNs, an optimized Zone-based Energy-Efficient Routing Protocol (OZEEP) [28] has been followed. OZEEP uses Genetic Fuzzy System (GFS) uses two steps for optimizing the clustering and the selection of $\mathrm{CH}$. Screening process is the initially done where all the nodes adopt the fuzzy system for electing some nodes for the $\mathrm{CH}$ role. This is based on their residual energy, distance from BS, neighbors, and mobility.

For trust estimations, the LTB-AODV protocol depends on IDS. So the performance is very poor when the random nodes' mobility and traffic patterns are in the picture. Many false alarm messages for reporting suspicious activities generated by the LTB-AODV results in the limitation of available bandwidth. Moreover, the drawback of existing routing protocols is that they consume high overhead controls in trust estimation and dissemination which leads to dead node formations and the routes become unstable.

Another scheme called Energy-aware Secure Routing with Trust (ESRT) was introduced by Adnan Ahmed et al. [29]. This paves way for a safe environment and avoids the misbehaving nodes. In order to come up with decisions for routing, the ESRT provides rust, energy, and hop counts. For balancing the energy consumption made by the trusted nodes during the routing using shorter distance, it is aided by the multi-facet routing strategy. Improvised results were exhibited by the ESRT scheme during simulation compared to the existing works. A source and a destination node will have only one route between them. The probability of call blocking has been increased by the single path routing protocols and also, there was a decrease in the total utilization of network. Apart from this, the end-to-end delay and the rate of packet loss have also increased due to single path protocol.

New multipath routing algorithms have been introduced for MANETs by Neha Bora et al. [30] which will merge the concept of a colony optimization along with Split Multipath Routing (SMR) protocol. Based on the swarm intelligence, the Ant colony optimization Split Multipath Routing (ACO-SMR) does its functionality especially on the Meta heuristic. The performances of the network like delay and ratio of packet delivery will be improved using the proposed algorithm when compared to the already existing routing algorithms

\section{DESIGN OF WORK}

\subsection{Overview}

There is a bunch of sensor nodes that are wireless present in the Wireless sensor network (WSN). They undergo exchange of data in a dynamic way among themselves without the liability on any destination node or WSN. The nodes are in mobility and are unable to be connected dynamically in an arbitrary way. The challenging design of sufficient routing protocols is due to the multi-hop, mobility, greater network size along with device heterogeneity, bandwidth and battery power constrains. Eventually, the connecting path of the source node to the destination nodes can be unstable and may switch down any time creating inconvenience for the ad hoc network. Previously, many single-path protocols especially for WSN have been proposed. Between the source and the destination there was only a single route is being used. The probability of preventing the call is increased due to single path routing protocols and there is a fall in the usage of the overall network. Adding on to this, the end-to-end delay as well as rate of packet loss could also be increased. For overcoming these issues, there comes an Optimized Dynamic secure multipath routing protocol for WSNs, merging the concept of ant colony optimization. This algorithm will enhance the performance of the network by overcoming issues like delay and packet delivery ratio as shown in Figure 1. 


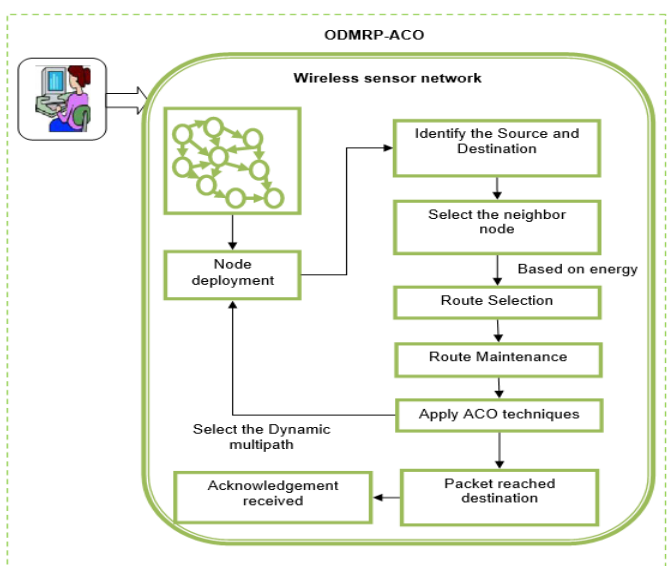

Figure 1. Overall proposed protocol

\subsection{Node deployment}

For monitoring the area around the network, the sensor nodes are embedded in the network environment. This will collect information from the sensor nodes. Various characteristics like transmission, sleep and monitoring are activated in the nodes and they are normally distributed. The source node transmits the data packets and there will be one neighbor node for receiving the data packets that the source sent. When the node's are registered, they are provided with Id, Names and other properties given by the server. The optimum path will be figures out by the sources and the key belonging to the intermediate nodes are gathered. For security reasons, all the communication between the nodes will be monitored by the network.

\subsection{Route selection}

Nodes will find their neighbors during the initialization of the network. Based on the transmission range, the residual energy and the level of information, the neighbors are selected. Only if a node has sufficient residual energy, a node will be selected as the neighbor and it should be present at comparatively a lower or similar depth level. Flooding is overcome by the depth threshold and the selection is based on the count of the node's neighbors. Each node is suggested to find its alive neighbors with a standard interval in this phase, so that the depth threshold could be adapted. With the help of the value of the depth threshold, and the data about residual energy of neighboring nodes, the data will be forwarded by the nodes.

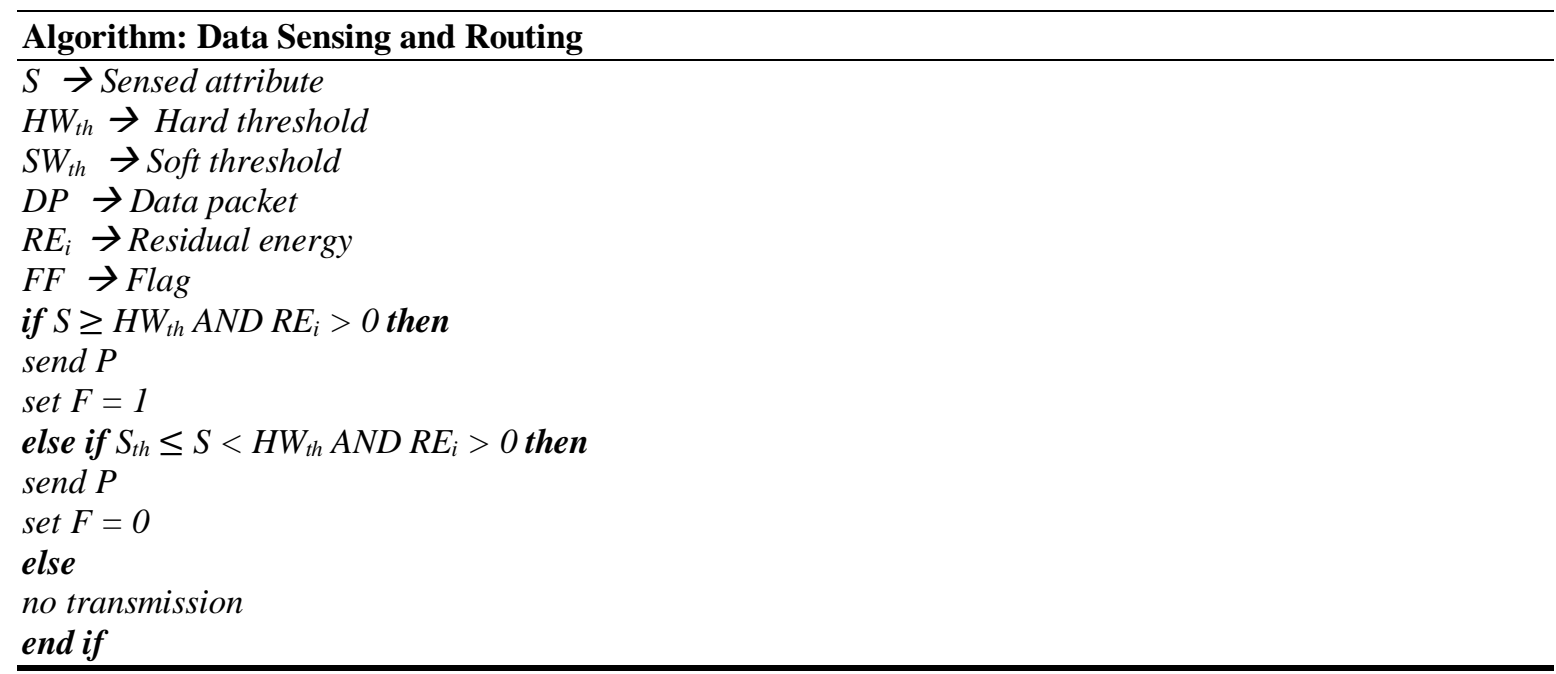

\subsection{Route maintained}

This shows how the nodes are connected to their neighbors in note of attaining self-stability and maximum energy. Messages can be exchanged between each node when the nodes are within the range of transmission. The data about connectivity, the one-hop neighbors' signal strength and the neighbor list are present in each node. The number of links (nodes) connected to it is represented by the $n$ which is the degree of it. 


\subsection{Ant colony optimization}

For determining the shortest path from the next node, the ACO depends on the ant colony cooperation behavior for obtaining the food source. The behavior of ants(social insects)that lives in a colony shows that they are interested in the matters of the colony. They have an peculiar and interesting way of finding the shortest distance between their source of food and their nest. Apart from these, the ants will secrete pheromone along the path. Likewise, in this routing process, the threshold value is compared with the destination sequence number obtained by the destination or the intermediate nodes. The control packets will be dropped off if the obtained value is greater than the threshold. The optimal path is determined by obtaining the largest destination sequence number that is lesser than the threshold value and with lower hop-count. Until any route error occurs or any break is seen, the same routing path will be followed. So, the overhead of the network will not occur as the same routing path is used and a less time for setting up of the route is taken. A brief routing scheme for the WSN is given by the Ant-Colony Optimization. This protocol consists of three phases quite similar to the other existing protocols. They are,
a) Route Discovery.
b) Route Maintenance.
c) Route Failure Handling

\subsection{Optimized dynamic secure multipath routing protocol - ACO}

The actual purpose of ACO is to dynamically determine the shortest path for data forwarding. On account of minimizing the energy consumption, cost, total energy consumption and finding the shortest path that is optimized along with selection of route dynamically, the ACO is being used. The ideal behavior of a real ant colony was the background cause for The Optimized Dynamic Secure Multipath Routing Protocol based Ant-Colony Optimization algorithm.

Below mentioned is the working procedure of ACO:

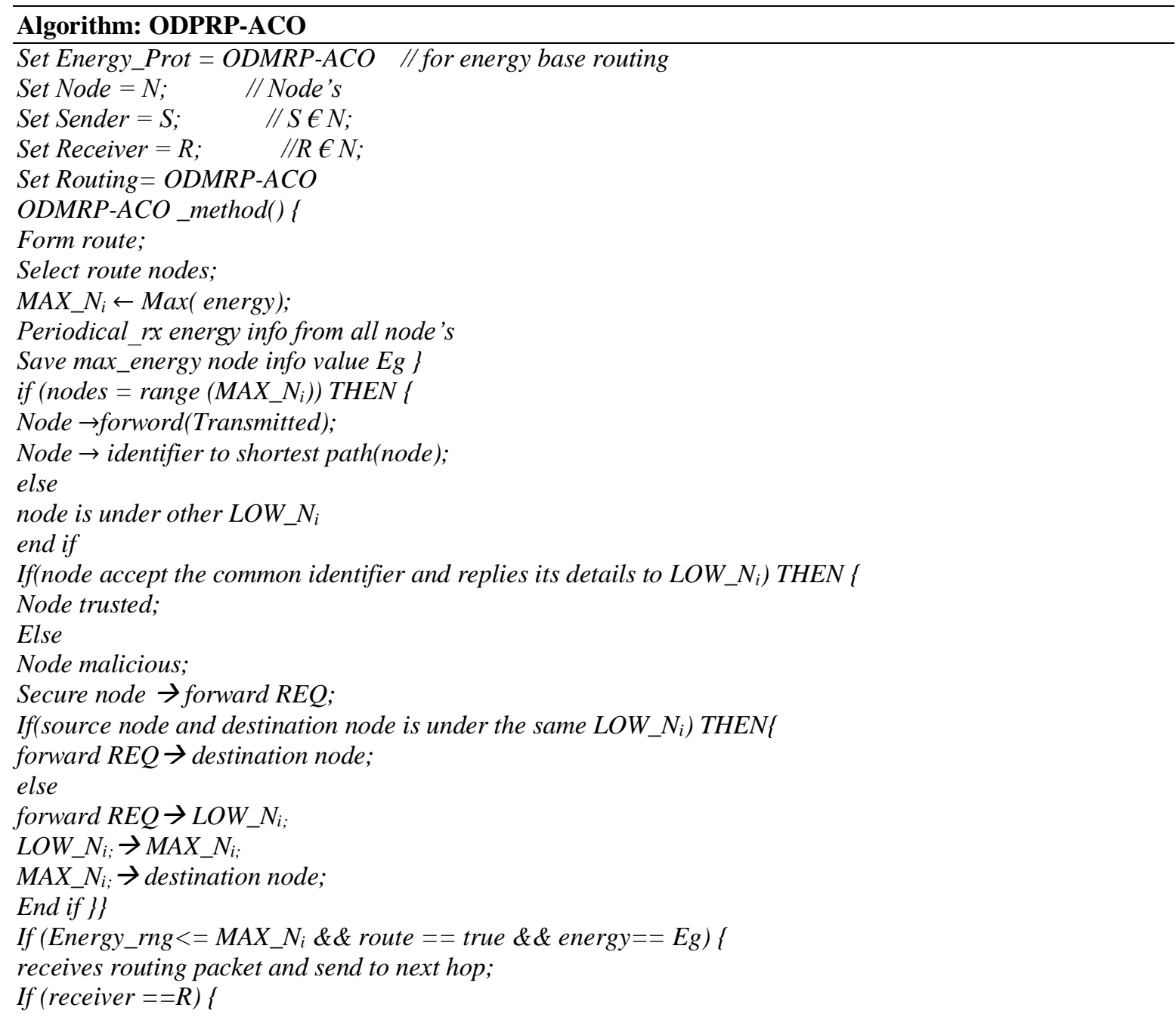


receives routing packet;

Send ackpkt to sender; \}

Else \{

receiver not exist ;\}

Else \{

node out of range or node is died or low energy \}

Terminate session; // that nodes id link failure because Low energy.

End if

Failure node $\rightarrow F N_{i} \quad$ // Dynamic path selection (Optimized path)

if $\left(\right.$ nodes $=$ range $\left(M A X \_N_{i}\right) \& \&$ route $==$ true $\& \&$ Distance $==$ Min $)$ THEN \{

Node $\rightarrow$ forward (Transmitted);

Node $\rightarrow$ identifier to shortest path (node);

else

node is under other $L O W_{-} N_{i}$

Source node $\rightarrow$ forward another neighbor node $\rightarrow$ next neighbor node;

Packet received $\rightarrow$ destination node

end if $\}$

end if $\}$

\subsection{Algorithm explanation}

Step 1: The WSN has a number of nodes(N) distributed in it.

Step 2: All the nodes defend same amount of energy during the start.

Step 3: Each node will have its own credentials like id, and it will transmit the same to all the nodes belonging to the network..

Step 4: The nodes that collect the id will check if the id is present in the memory already.

Step 5: If it is already there, then data discarding occurs.

Step 6: Or if it was not present, then the higher outstanding energy node is selected with the distance path that is the shortest.

Step 7: If the residual energy and the distance are to be the same, then a higher timestamp value will be used during packet transmission.

Step 8: Until the destination is known, the information related to the location should be maintained.

Step 9: Failure of sending the packets may occur if the energy is low for the node. Due to this data loss can occur.

Step 10: Now like the ants' way of finding the optimized path from source to destination, the ODMPR-ACO is used in the process.

Step 11: Maximum energy is tried to be attained while selecting and sending of packets.

Step 12: Confirmation of the data received at the destination node.

Step 13: If the data is received successfully, then an acknowledgment is sent to the source node regarding this. The optimized dynamic multipath selecting process is explained Figure 2 which is used for data sharing and forwarding. This is closed on the basis of energy, distance and minimum hub count.

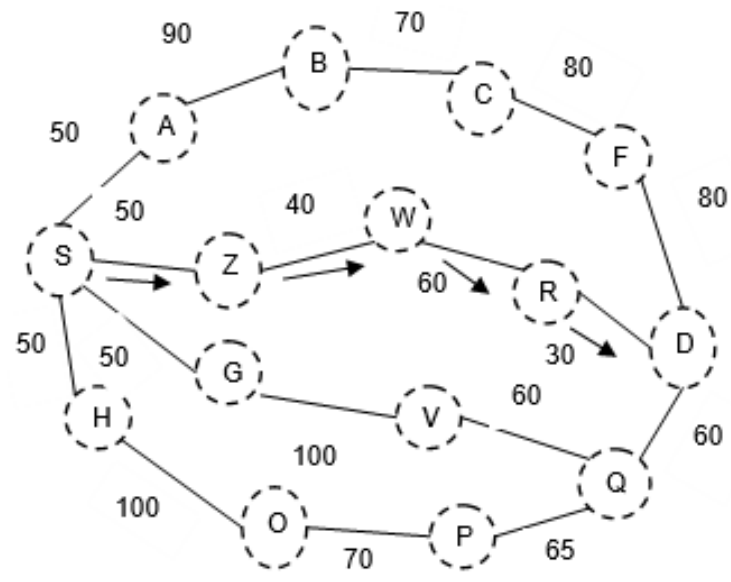

Figure 2. Effects of selecting different switching under dynamic condition 


\subsection{Ant Colony Optimization using Traveling Shortest Path Problem}

The matrix of the shortest distances (dimension $\mathrm{n}^{*} \mathrm{n}$ ) is considered to be $\mathrm{C}$, where the number of nodes in the graph $\mathrm{G}$ is represented by $\mathrm{n}$. The matrix elements will be showing the available shortest distances between all the node pairs $(\mathrm{i}, \mathrm{j}), i, j=1,2, \ldots, n$.

Depending on the fact if the route from node $i$ to node $j$ is realized $\left(x_{i j}=1\right)$ or not $\left(x_{i t}=0\right)$, the formulation of ACO occurs in the programming binary category where the variables can take the values either 0 or 1 . The TSP is formulated to assign numbers from 1 to $\mathrm{n}$ to the nodes along with few more variables $u_{i}$, corresponding to the numbering order for the nodes present in the path. This is how the mathematical formulation of TSP occurs. Since the sub-path apart from node 1 should not have a feasible assignment of the

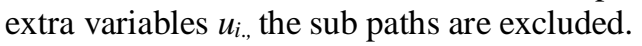

$$
\min \sum_{i=1}^{n} \sum_{j=1}^{n} c_{i j} x_{i j}
$$

Subject to

$$
\begin{aligned}
& \sum_{i=1}^{n} x_{i j}=1 \quad i=1,2, \ldots n, \quad i \neq j \\
& \sum_{j=1}^{n} x_{i j}=1 \quad j=1,2, \ldots n \quad i \neq j \\
& u_{i}-n x_{i j} \leq n-1 \quad i, j=1,2,3, \ldots n \quad i \neq j \\
& x_{i j} \in\{0,1\} \quad i, j=1,2,3, \ldots n \quad i \neq j
\end{aligned}
$$

The idea behind this is to come up with the organized nature of the ant colony behavior which is called as the ant colony algorithm. In the case of foraging ants, they keep track on finding the pheromone, which gives the shorter path. If the pheromone is unattended then it will cause the other ants to be conscious of the paths and will find the shortest path for task completion.

For example, if at time $\mathrm{t}$, ant $\mathrm{k}$ is transferred from node to node $\mathrm{J}$, then the equation of the transition probability is described as follows:

$$
p_{i j}^{k}(t)=\left\{\begin{array}{c}
\frac{\left[A_{i j}(t)\right]^{\alpha} *\left[B_{i j}(t)\right]^{\beta}}{\sum_{s \in \text { allow } k}\left[A_{i s}(t)\right]^{\alpha} *\left[B_{i s}(t)\right]^{\beta}} j \in \text { allowed } k \\
0 \quad \text { otherwise }
\end{array}\right.
$$

$A_{i j}(t) \rightarrow$ Denote the transition's attractiveness previously.

$B_{i j}(t) \rightarrow$ Transition attractiveness is added for ants.

$\mathrm{S} \rightarrow$ Denotes the set of nodes that are pointing to I excluding the finally visited point before I.

$\alpha, \beta \rightarrow$ Parameters found by simulation.

The heuristic information will be damaged when the pheromones are deposited in a large amount and the path finding process will still be carried out by the ants. Therefore, the pheromone update rule should be updated constantly. The classical ant colony algorithm is mentioned below.

$$
\begin{aligned}
& A_{i j}(t+n)=(1-p) \cdot A_{i j}(t)+\Delta A_{i j}(t) \\
& \Delta A_{i j}(t)=\sum_{k=1}^{n} \Delta A_{i j}^{k}(t)
\end{aligned}
$$

Wherein, $\rho \in(0,1)$ for the retention factor pheromone, under $\triangle A_{i j}(t)$ is the path, the sum of the newly added information element, $\triangle \mathrm{A}_{\mathrm{ij}}(\mathrm{t})$ at time $\mathrm{k}$ ant $(\mathrm{t}, \mathrm{t}+\mathrm{n})$ remaining on this path is calculated.

There are various issues to be considered in Network routing optimization as they differ from the conventional planning of paths. Some of the issues are, delay in data presence, delayed Throughput and other 
issues during transmission between the routing nodes. So, this paper explains issues like delay in lifespan of the network, the throughput, bandwidth and energy consumption paths. The delay and their impacts are taken into account when the state transition process occurs. An improved formula for state transition is as follows:

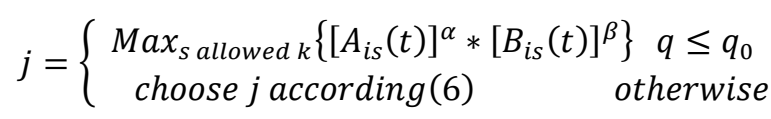

Here, $\mathrm{q}_{0}$ between $0-1$, and $\mathrm{q}$ is a random number that follows uniform distribution between $0-1$. nij will no more be the function of the inverse path. However, the delay and delay jitter and a countdown function is improved. Calculating the finish time of the exit task will give you the distance of length. Make span is the other term for Distance of length.

\section{Distance of Length Ratio (DLR)}

DLR is considered to be the ratio in Distance of length to the optimized path

$$
\mathrm{DLR}=\frac{\mathrm{DL}}{\text { optimizied Path }}
$$

\section{Speedup (SP)}

The ratio of time taken for solving the issue in a single processing element (sequential execution) to the time taken by solving in a parallel computer (parallel execution) is called the speedup.

$$
\mathrm{SP}=\frac{\text { Sequential execution time }}{\text { Parallel execution time }}
$$

\section{Energy Efficiency (EEFF)}

The ratio of speedup to the processing element count is known as the Energy Efficiency (EEFF). It measures the fraction of time that the processing element was used effectively. How well the resources are utilized for the program execution is the ultimate reason behind this. Cost - The parallel execution time and how many nodes were created contributes to the cost.

$$
\mathrm{EEFF}=\frac{\mathrm{SP}}{\text { Number of Processing elements }}
$$

Cost

The parallel execution time and how many nodes were created contributes to the cost.

$$
\text { Cost }=\text { No. of nodes } \times \text { parallel execution time }
$$

\subsection{Advantages}

a) Near optimization is obtained by the proposed scheme in various network configurations.

b) Lifespan of the sensor networks is increased through duty scheduling in wireless sensor networks.

c) An adaptive mechanism during traffic and routing of multipath is provided

d) Depending on the passive and active information monitoring and retrieval

e) Focus on the reduced delay and consumption of energy in the WSN A protocol that is energy efficient.

\section{RESULTS AND DISCUSSION}

For checking the performance of the proposed scheme, the NS2 simulator was used. The obtained results showed that there was better efficiency and improved performance when various metrics were considered when distinguished between the other existing approaches from the literature. The simulation parameters are shown in Table 1 and this is used for the protocol design. The result of simulation is shown in Figure 3. This is the best shortest path that was selected on the basis of energy and the distance for data forwarding. 
Table 1. Simulation parameters

\begin{tabular}{ll}
\hline \multicolumn{1}{c}{ Parameter } & Value \\
\hline Simulator & NS-2(v2.34) \\
Simulation Landscape & $2500^{*} 1000$ \\
No. of. nodes & 50 \\
Node Type & Heterogeneous \\
Transmission Range & $250 \mathrm{~m}$ \\
Node Energy & $100 \mathrm{~J}$ \\
Packet size & $1000 \mathrm{bits}$ \\
Transmission Range & $100 \mathrm{Kbytes}$ \\
Antenna Type & Omni directional \\
Mobility Models Communication & Random-waypoint $(0-30 \mathrm{~m} / \mathrm{s})$ \\
Radio Frequency & $850-950 \mathrm{MHz}$ \\
Routing Protocol & ODMRP-ACO \\
MAC Protocol & IEEE 802.11 \\
Background Data Traffic & CBR \\
Packet Interval & $0.01 \mathrm{sec}$ \\
\hline
\end{tabular}

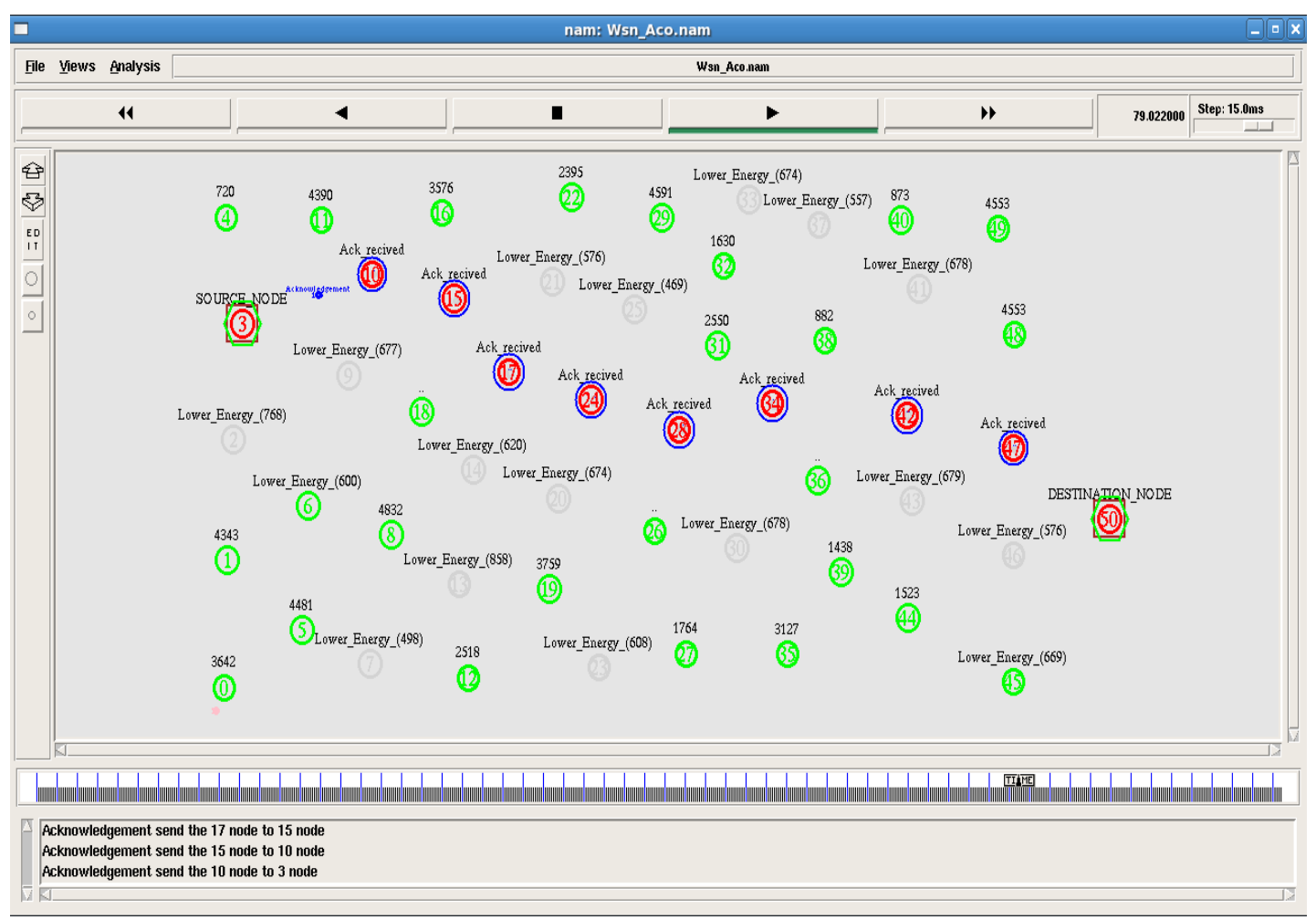

Figure 3. Simulation result diagram

Energy Consumption: The amount of energy that is utilized during the communication and data processing is called as energy consumption.

Figure 4 depicts the energy consumption variation seen in the network for the nodes range from 50500 . The usage of energy for that network will reach a maximum of $15.10 \mathrm{~J}$ when the node count is 50 . In the process of increasing the node count from 50 nodes to 100 nodes in the same network, the consumption of energy is observed to fall down. Again, by increasing the count from 100 to 200, there is an increase seen in the minimum energy consumption which is very progressive. When the count crosses 400 , there is a decrease in the minimum energy consumption for that network. Exactly at 500, $8.04 \mathrm{~J}$ is the value recorded for the energy consumption which is comparatively a lower value. 


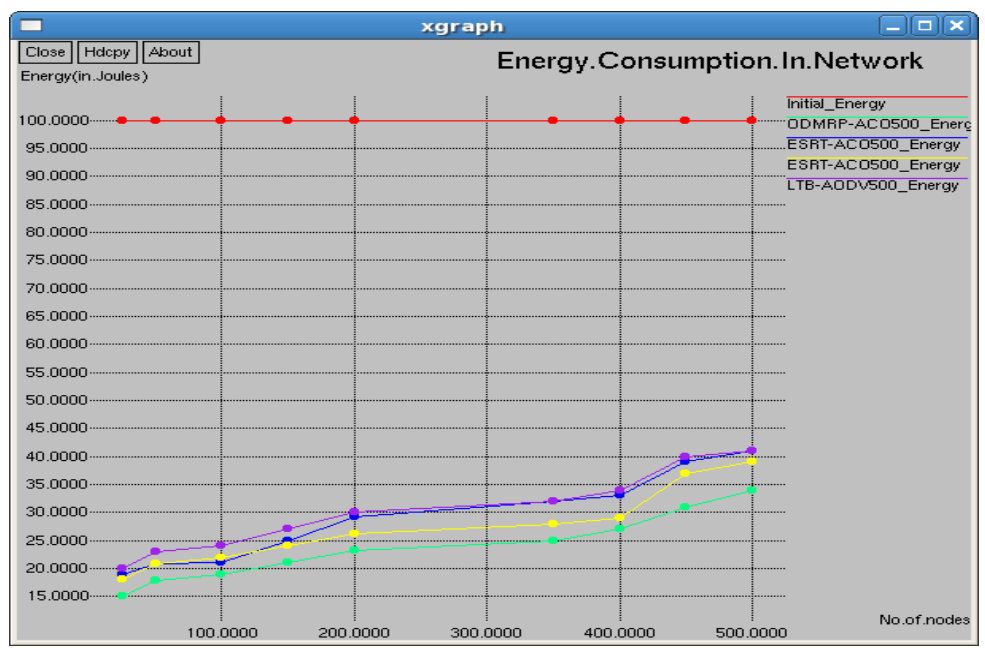

Figure 4. Energy consumption

There are various existing techniques like ESRT-ACO, SMR-ACO, LTB-AODV and ODMRP-ACO methods. Used for finding the average energy consumption. Of all these, the ODMRP-ACO technique gives considerably a greater output than the other techniques.

Packet delivery ratio: The number of packets that a node is capable of transmitting at a desired time is called the packet delivery ratio. When compared with the usual WSN, the packet delivery ratio seems better in energy properties as well as cutting down of duplication. The graph of comparison is shown in the Figure 4:

Packet delivery ratio: The ratio between the number of packets that were delivered to the destination is known as the packet delivery ratio.

$$
\sum \text { Number of packets received/ } \sum \text { number of packets send }
$$

The higher the packet delivery ratio, the greater the protocol's performance will be shown in Figure 5.

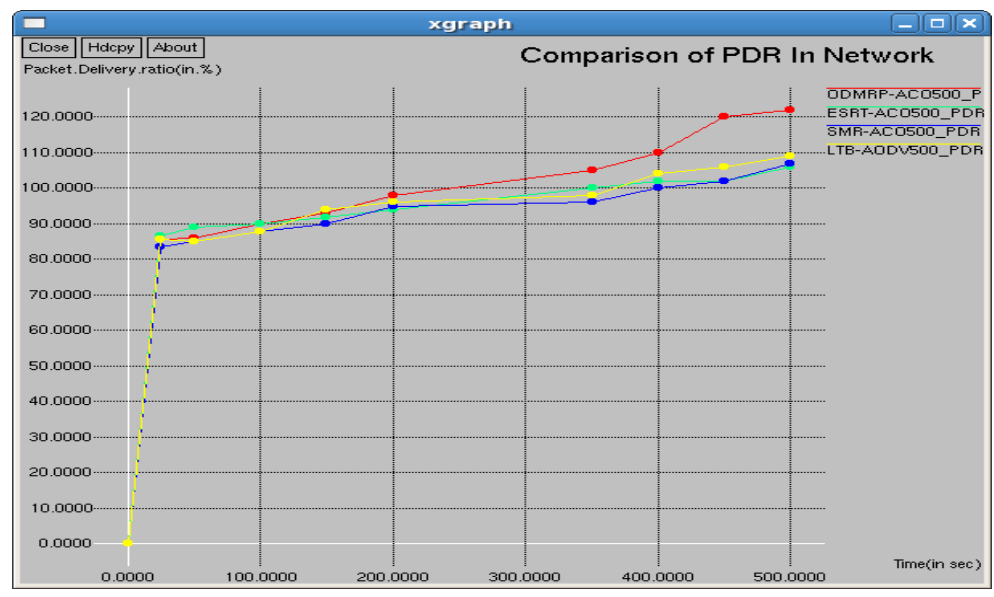

Figure 5. Packet delivery ratio

When the number of nodes in the network is 50, the detection mechanism that was proposed by the author exhibits a packet delivery of $95 \%$ and when it is 100 nodes, it is reduced to $89 \%$. The packet rate is observed to be high for small node range in the network. When the node count is 200, again there is a decrease in the packet ratio where the curve is reduced to $85 \%$. When it is 400 nodes in the network, the curve is seen to reach $87 \%$. Towards 500 nodes in the network, there is reduces of $1 \%$ becoming $84 \%$ of packet delivery ratio. So, even when the value reduces, it is better than the existing protocols. 
End to end delay: The time taken for the delay in receiving the re-transmitted packet in each node is called the end to end delay. After comparison, the result shows that the delay occurred was comparatively lower than the already present in the Wireless Sensor Network which is shown in the Figure 6.

Average end-to-end delay: This is the average time consumed by the data packet for reaching the destination node.

$$
\sum(\text { Arrive time }- \text { sent time }) / \sum \text { Nomber of connections }
$$

The performance of the protocol and the end to end delay are inversely proportional to each other. Figure 6 shows the end to end delay in the approach. If the network has 50 nodes, then the end to end delay would be at least $0.26 \mathrm{msec}$ where the curve moves from 0 to 50 . Then it progresses and reaches $0.31 \mathrm{msec}$ for a network with 200 nodes. When the node count reaches 400, the curve attains a value of $0.39 \mathrm{msec}$. At the max nodes of 500 in the network, the curve attains $0.53 \mathrm{msec}$ of end to end delay. Here it is clearly evident that for lesser node count in the network, the delay is also minimum.

Throughput: The average number of packets being transferred by a node is known as throughput. The throughput is compared alongside the usual WSN and the improved WSN. The final results of the improved WSN come to be $86 \%$ better than the existing WSN. Figure 7 shows the performance comparison.

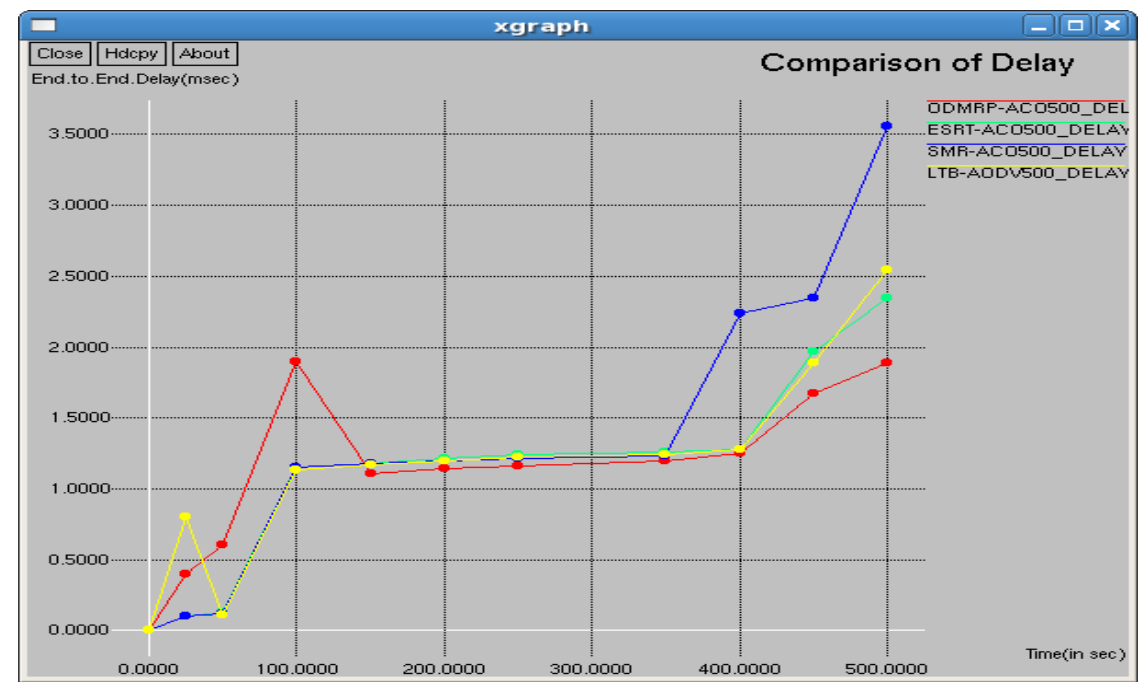

Figure 6. End-to end delay

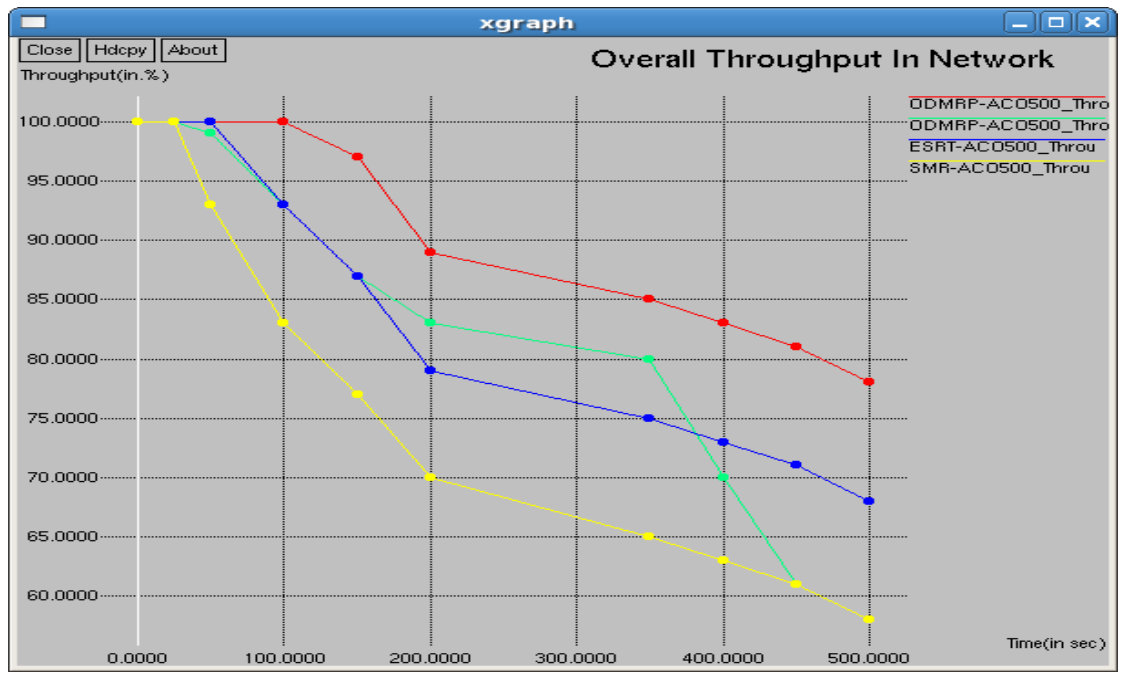

Figure 7. Throughput 
A $90 \%$ throughput has been reached when analyzing the improvement performance. When the network had 50 nodes in the start, the throughput went to $99 \%$ and a very slight increase was observed when the node count was increased from 50 to 100. Then there was a decrease from $98 \%$ to $94 \%$ when the network had 200 nodes. The throughput performance rate went up to $84 \%$ from $81 \%$ when there was an increase in the network size which had 400 nodes. For a node size of 500, the curve has shown an increase from $76 \%$ to $78 \%$.

The comparison between the Network lifetime analysis with the already present techniques like Optimized dynamic secure multipath routing protocol-Ant colony optimization(ODMPR-ACO), Energy-aware and Secure Routing with Trust (ESRT-ACO), Split multipath routing protocol-Ant colony Optimization(SMRACO) and Light Weight Trust Based routing protocol (TLB-AODV) methods are shown in Figure 8. A better output is obtained using the ODMPR-ACO technique.

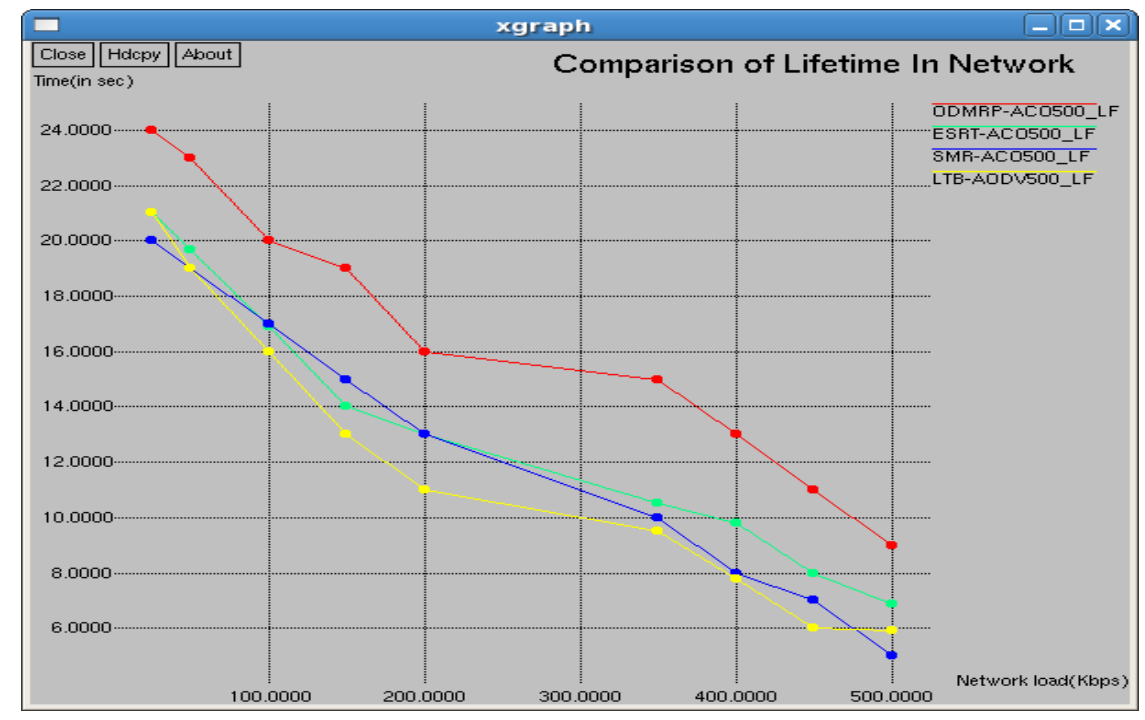

Figure 8. Network lifetime

The packet loss comparison with the existing techniques like Optimized dynamic secure multipath routing protocol-Ant colony optimization(ODMPR-ACO), Energy-aware and Secure Routing with Trust (ESRT-ACO), Split multipath routing protocol-Ant colony Optimization(SMR-ACO) and Light Weight Trust Based routing protocol (TLB-AODV) are shown in Figure 9. A better outcome is seen by the use of ODMPRACO method [31][32][33].

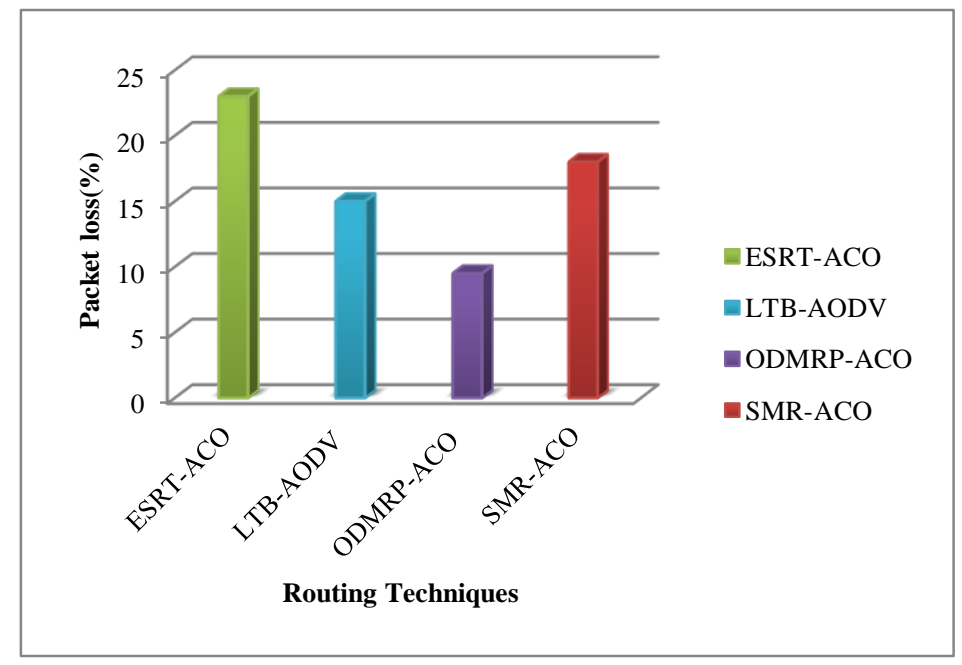

Figure 9. Packets loss 
Figure 10 depicts the performance comparison of Routing techniques with various existing techniques such as Optimized dynamic secure multipath routing protocol-Ant colony optimization(ODMPR-ACO), Energy-aware and Secure Routing with Trust (ESRT-ACO), Split multipath routing protocol-Ant colony Optimization(SMR-ACO) and Light Weight Trust Based routing protocol (TLB-AODV). When the ODMPRACO technique is used, it shows high performance and better outputs when compared to the existing techniques.

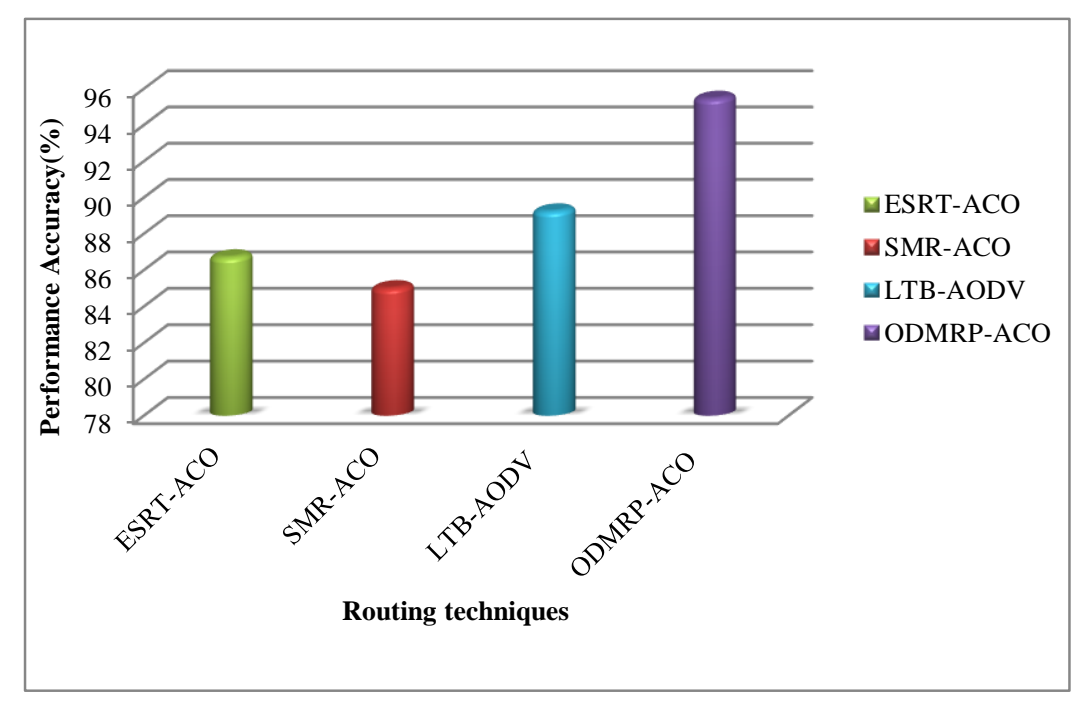

Figure 10. Comparison of routing techniques

\section{CONCLUSION}

The main idea of this algorithm is to improve the energy and thereby extend the network span of a WSN embedded in the environment. For increasing the battery life and reducing the energy consumption, the Optimized Dynamic secure multipath routing protocol approach is one among the suggestive methods. For transmitting data with a secure and promising QoS in the Wireless Sensor Networks (WSNs), the routing optimization technique has been introduced. It was simulated in NS2 simulation software environment and follows the Ant Colony Optimization (ACO). Better performance in aspects of throughput, Energy consumption and Packet Delivery Ratio (PDR), has been recorded during simulation. So, it is proved that the ACO possess the capability of optimizing the routing algorithms which facilitates the multimedia applications over WSNs.

\section{REFERENCES}

[1] Mahmoud M. Elkholy, Mohamed A. Elhameed. Minimization of Starting Energy Loss of Three Phase Induction Motors based on Particle Swarm Optimization and Neuro Fuzzy Network. International Journal of Power Electronics and Drive System (IJPEDS), Vol. 7, No. 4, December 2016, pp. 1038 1048.

[2] Mahmoud M. Elkholy, Mohammed A. Elhameed. Braking of Three Phase Induction Motors by Controlling Applied Voltage and Frequency Based on Particle Swarm Optimization Technique. International Journal of Power Electronics and DriveSystem (IJPEDS). 2015; 5(4): 520-528.

[3] Ahmed Zubair, Aman Abdulla Tanvir, Md. Mehedi Hasan. Optimal Planning of Standalone Solar-Wind-Diesel Hybrid Energy System for a Coastal Area of Bangladesh. International Journal of Electrical and Computer Engineering (IJECE) Vol.2, No.6, December 2012, pp. 731 738 ISSN: 2088-8708.

[4] Anand M, V. Mathivananr. Energy Management in Current Fed Switched Inverter Based Propulsion Motor for Marine Application. Indonesian. Journal of Electrical Engineering and Computer Science Vol. 9, No. 2, February 2018, pp. 282 285 ISSN: 2502-4752, DOI: 10.11591/ijeecs.v9.

[5] S. Rohit, et al., Pulse Density Modulation Flyback Converter for LED Automotive Lighting, Indonesian Journal of Electrical Engineering and Computer Science, vol/issue: 8(1), 2017.

[6] Suresh Kumar J, Babu Raj E. Genetic algorithm based multicast routing in wireless sensor networks - A research framework. International Journal of Engineering and Innovative Technology (IJEIT), 2012; 2(6): 240-246.

[7] Rama Sundari Battula, Khanna OS. Geographic routing protocols for wireless sensor networks: A review. International Journal of Engineering and Innovative Technology (IJEIT), 2013; 2(12): 39-42. 
[8] Muhammad Fuad, Muhammad Iqbal, Mamat Rahmat, Heru Sukoco, Husin Alatas. Performance Analysis of ZigBee Mesh WSN in CarbonMonoxide Gas Monitoring System. TELKOMNIKA Indonesian Journal of Electrical Engineering, 2015; 15(3): 576-583.

[9] Abdul Latiff NM. A Training Monitoring System for Cyclist Based on Wireless Sensor Networks. Indonesian Journal of Electrical Engineering and Computer Science. 2017; 6(1): 80-87.

[10] Anand Nayyar, Rajeshwar Singh. Ant Colony Optimization (ACO) based Routing Protocols for Wireless Sensor Networks (WSN): A Survey. (IJACSA) International Journal of Advanced Computer Science and Applications, 2017; $8(2)$.

[11] Nandini G, Anitha J. Performance Chronicles of Multicast Routing Protocol in Wireless Sensor Network. (IJACSA) International Journal of Advanced Computer Science and Applications. 2017; 8 (9).

[12] Hao J, Duan G, Zhang B, Li C. An Energy-Efficient On-Demand Multicast Routing Protocol for Wireless Ad Hoc and Sensor Networks. IEEE Globecom-Wireless Networking Symposium. 2013.

[13] Liu X. Atypical Hierarchical Routing Protocols for Wireless Sensor Networks: A Review. IEEE Sensors Journal. 2015; 15 (10):5372-5383.

[14] Maddali BK. Core network supported multicast routing protocol for wireless sensor networks. IET Wireless Sensor Systems. 2015.

[15] Kumar D. Performance analysis of energy efficient clustering protocols for maximising lifetime of wireless sensor networks. IET Wireless Sensor System. 2017; 4 (1): 9-16.

[16] Velmani R, Kaarthick B. An Efficient Cluster-Tree Based Data Collection Scheme for Large Mobile Wireless Sensor Networks. IEEE Sensor Journal. 2015; 15 (4): 2377-2390.

[17] Anand Motwani, Vimal Dhote. Optimized AODV Routing for Effective Attack Security in Wireless Sensor Networks. International Journal of Electrical, Electronics and Computer Engineering. 2016; 5(1):33-40.

[18] Seema Pahal, Kusum Dalal. A Review Paper on Routing Protocols in Wireless Sensor Networks. International Journal of Recent Trends in Engineering \& Research (IJRTER). 2016; 2(3).

[19] Wang CF, Shih JD, Pan BH, Wu TY. A Network Lifetime Enhancement Method for Sink Relocation and Its Analysis in Wireless Sensor Networks. IEEE Sensors Journal. 2014; 14(6); 1932-1943.

[20] Davut Incebacak, Ruken Zilan, Bulent Tavli, Jose M. Barcelo-Ordinas, Jorge Garcia-Vidal. Optimal data compression for lifetime maximization in wireless sensor networks operating in stealth mode. Ad Hoc Networks. 2015; 24: 134-147.

[21] Keontaek Lee, Hak-Jin Kim, Sunju Park, Seungjae Han. Satisfying the target network lifetime in wireless sensor networks. Computer Networks. 2014; 65: 41-55.

[22] Subir Halder, Sipra Das Bit. Enhancement of wireless sensor network lifetime by deploying heterogeneous nodes. Journal of Network and Computer Applications. 2014; 38: 106-124.

[23] Sheikholeslami A, Ghaderi M, Pishro-Nik H, Goeckel D. Energy-Efficient Routing in Wireless Networks in the Presence of Jamming. IEEE Transactions on Wireless Communications, 2016; 15(10): 6828-6842.

[24] Emansa Hasri Putra, Risanuri Hidayat, Widyawan Widyawan Wayan Mustika. A Routing Optimization Based on Ant Colony for Wireless Multimedia Sensor Networks (WMSNs). International Journal of Intelligent Engineering and Systems, 2016; 9(4).

[25] Pantazis, Nikolaos, Stefanos A. Nikolidakis, Dimitrios D. Vergados. Energy-efficient routing protocols in wireless sensor networks: A survey. Communications Surveys \& Tutorials, IEEE, 2013: 551-591.

[26] Putra EH, Hidayat R, Widyawan, Mustika IW. Cross-Layer Design of Wireless Multimedia Sensor Network Based on IEEE 802.11e EDCA and H.264/SVC. International Conference on Science in Information Technology (ICSI Tech).2016.

[27] Attea BAA, Khalil EA, Cosar A. Multi-objective evolutionary routing protocol for efficient coverage in mobile sensor networks. Soft Computing, 2015; 19(10): 2983-2995.

[28] Srivastava JR, Sudarshan TSB. A genetic fuzzy system based optimized zone based energy efficient routing protocol for mobile sensor networks (OZEEP). Applied Soft Computing Journal, 2015; 37: 863-886.

[29] Adnan Ahmed, Kamalrulnizam Abu Bakar, Muhammad Ibrahim Channa, Abdul Waheed Khan, Khalid Haseeb. Energy-aware and secure routing with trust for disaster response wireless sensor network. Springer Science + Business Media New York. 2015.

[30] Zhang C, Zhu X, Song Y, Fang Y. A formal study of trust based routing in wireless ad hoc networks. I.E. Proceedings INFOCOM 14-19 March. San Diego, CA: IEEE. 2010: 1-9.

[31] Fernandez-Gago MC, Roman R, Lopez J. A survey on the applicability of trust management systems for wireless sensor networks. Third IEEE International Workshop on Security, Privacy and Trust in Pervasive and Ubiquitous Computing, SECPerU. 19- 21 July. Istanbul: IEEE. 2007: 25-30.

[32] Neha Bora, Jigyasha Arora and Nitin Arora. Ant colony based optimization algorithm for the Mobile ad hoc Network Routing Problemsbased on SMR Protocol (ACO-SMR). International Journal of Application or Innovation in Engineering \& Management (IJAIEM). 2013; 2(6).

[33] Adnan Ahmed. Energy-aware and secure routing with trust for disaster response wireless sensor network. Peer-toPeer Netw. Appl. \# Springer Science+Business Media New York. 2015. 